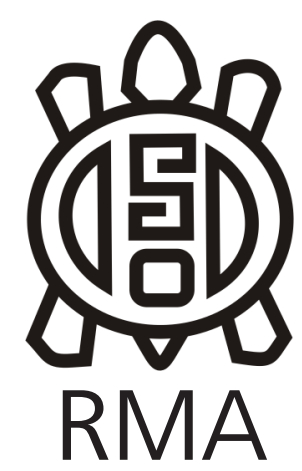

Dossier

\title{
Investigaciones geoarqueológicas de fuentes líticas en los Andes argentinos: génesis de las rocas, costos de accesibilidad y sistemas de producción lítica
}

\author{
Geoarchaeological research on lithic raw material sources in \\ the Argentine Andes: geologic formation, accessibility costs and \\ lithic production systems
}

\author{
Silvina Castro*, Lucía Yebra**, Valeria Cortegoso*, \\ Gustavo Lucero***, Víctor Durán*, Erik Marsh*; Diego Winocur****
}

\begin{abstract}
*CONICET, Universidad Nacional de Cuyo, Facultad de Ciencias Exactas y Naturales, Laboratorio de Paleoecología Humana. E-mail: silvinacastro2015@gmail.com,vcortegoso@gmail.com, duranvic@gmail.com, erik.marsh@gmail.com

**ANPCyT, Universidad Nacional de Cuyo, Facultad de Ciencias Exactas y Naturales, Laboratorio de Paleoecología Humana. E-mail:yebralucia@hotmail.com ***Universidad Nacional de Cuyo, Facultad de Ciencias Exactas y Naturales, Laboratorio de Paleoecología Humana, Argentina. Departamento de Antropología, Facultad de Ciencias Sociales y Humanidades, Universidad Católica de Temuco, Chile E-mail: glucero18@gmail.com

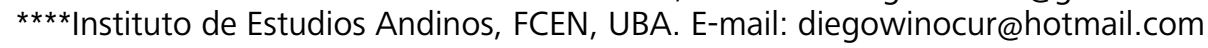

\begin{abstract}
Resumen
Se presenta una síntesis de resultados de estudios geoarqueológicos de fuentes y canteras líticas ubicadas en

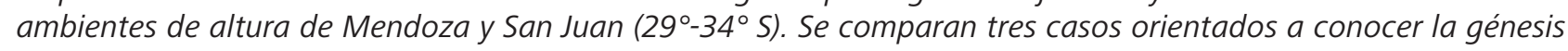
de las rocas, su disponibilidad como recurso lítico, los modos de aprovisionamiento y los variables sistemas de producción. Los trabajos se basan en relevamientos interdisciplinarios de campo y la aplicación de diversos métodos de análisis petrográficos, geoquímicos y de SIG. En San Juan (29ㅇ) se identificaron diez fuentes primarias y secundarias que fueron explotadas diferencialmente durante ca. 8500 años. En el noroeste de Mendoza (32 $\left.{ }^{\circ} \mathrm{S}\right)$ se constató la explotación como cantera lítica de los bosques petrificados de Darwin y de la formación Los Colorados. En el centro de Mendoza (34ㅇ) se identificaron dos señales químicas locales de obsidiana. Se enfatiza el potencial de estos estudios para indagar sobre cambios y continuidades en estrategias humanas de explotación de ambientes de altura del centro occidente de Argentina.
\end{abstract}

Palabras clave: fuentes y canteras líticas; geoarqueología; ambientes andinos.

\begin{abstract}
This article presents a synthesis of geoarchaeological studies of lithic raw material sources and quarries from three high-altitude areas in Mendoza and San Juan (29-34º S). These studies sought to identify the origin of the geological outcrops, their availability to humans as lithic resource, means of provisioning, and variable production systems. The studies rely on interdisciplinary field surveys and incorporate a variety of methods such as petrography, geochemistry, and Geographic Information Systems (GIS). In the San Juan province (295), ten primary and secondary lithic sources were differentially exploited for around 8500 years. In the northwest of Mendoza province (32 ${ }^{\circ}$ S), Darwin's petrified forest and the Los Colorados formation were used as quarries. In the center of Mendoza province (34 $\left.{ }^{\circ} \mathrm{S}\right)$, two local obsidian geochemical signatures were identified. We emphasize the potential of this kind of studies to evaluate continuities and changes in human mobility strategies in high-altitude areas in central western Argentina.
\end{abstract}

Keywords: lithic sources and quarries; geoarchaeology; Andean environments.

Uno de los planteos frecuentes en los trabajos de tecnología está vinculado con la disponibilidad regional y la procedencia de los recursos líticos. Esta información inicial sobre la disponibilidad de rocas para la talla permite efectuar hipótesis o inferencias sobre diversos aspectos vinculados a las relaciones entre localidades geográficas, el rango de acción y grado de movilidad de los grupos, la interacción entre poblaciones, los sistemas de producción lítica y las estrategias de aprovisionamiento, entre otros (Ericson, 1984; Ingbar, 1994; Nelson, 1991; entre otros). 
En la última década se han desarrollado diversos proyectos con el objetivo de identificar y estudiar sistemáticamente fuentes y canteras en ambientes de altura de las provincias de Mendoza y San Juan. Los trabajos incluyeron relevamientos geológicos y arqueológicos, geoquímica de obsidianas, análisis petrográficos, Sistemas de Información Geográfica (SIG) y análisis tecnológico del registro lítico (Castro et al., 2014, Cortegoso et al., 2014, 2017, De Francesco et al., 2018, Giesso et al., 2011, Lucero, 2015, Lucero et al. 2017). En este trabajo se comparan tres casos de estudio con diferentes niveles de avance en su investigación, se sintetizan los principales resultados y se proyecta la posibilidad de estudios futuros.

Las áreas de trabajo se concentran en espacios de altura ubicados en tres franjas latitudinales entre los $29^{\circ}$ y $34^{\circ}$ de latitud Sur: 1) cuenca alta del río de Las Taguas en el noroeste de San Juan $\left(29^{\circ} \mathrm{S}\right), 2$ ) Bosque de Darwin y Formación Los Colorados en el noroeste de Mendoza $\left(32^{\circ} \mathrm{S}\right)$ y 3 ) Laguna del Diamante en el centro oeste de Mendoza $\left(34^{\circ} \mathrm{S}\right)$ (Figura 1). Estos espacios se caracterizan por una topografía abrupta y un clima desértico de altura. En verano ofrecen recursos hídricos y abundante biomasa vegetal y animal (Lama guanicoe, por ejemplo). En invierno, la dinámica ambiental condiciona el asentamiento y la circulación humana debido a las bajas temperaturas y, en la mayor parte de los casos, a la acumulación nívea. Son ambientes complementarios con otros ubicados a menor altura. Tal complementariedad favorece una explotación humana dinámica de diferentes pisos altitudinales, comportamiento característico en espacios áridos y ecológicamente inestables (Aldenderfer, 2008).

A partir del análisis de cambios en las estrategias tecnológicas se están estudiando procesos de diversificación en la base económica en el noroeste de San Juan y Mendoza (Cortegoso, 2005, 2006, Cortegoso et al., 2014, Durán et al., 2006, Lucero et al., 2017). Conocer la disponibilidad de materias primas líticas en estas regiones mediante el estudio de fuentes y sitios de funcionalidades diversas ha permitido orientar las preguntas sobre cambios en la modalidad de abastecimiento registradas a lo largo del Holoceno e implementadas por sociedades con distintos sistemas de subsistencia y movilidad.

\section{Consideraciones metodológicas generales}

En primer lugar se confeccionó una base regional de recursos líticos para cada área (sensu Ericson, 1984). Todos los trabajos de campo se realizaron con el geólogo Dr. Diego Winocur, lo que permitió precisar la caracterización geológica de los afloramientos, identificar espacios con disponibilidad de recursos aptos para la talla lítica y su correcta distinción como afloramientos primarios o dispersiones secundarias y como "fuentes con taller" o "canteras potenciales" (sensu Nami, 1992).

El proceso de talla puede ocurrir a lo largo de una región y

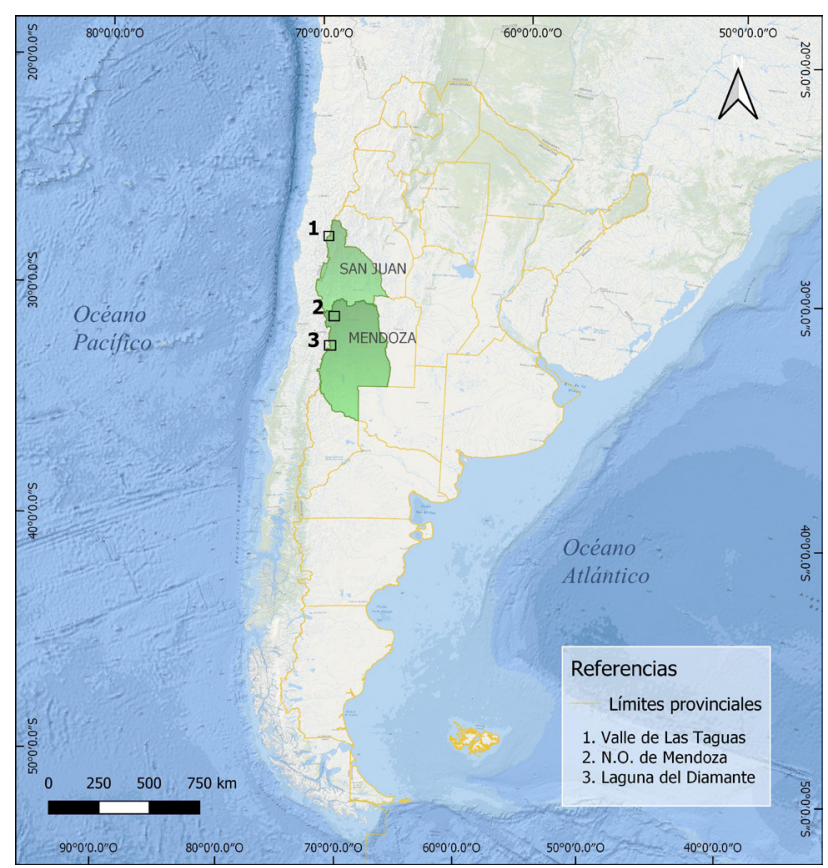

Figura 1. Localización de las áreas de estudio.

Figure 1. Location of the study areas.

las etapas de manufactura pueden ser efectuadas en uno o varios sitios (Ericson, 1984). En consecuencia, los trabajos que se presentan incluyeron tres escalas espaciales de análisis (sensu Dincauze, 2000). En la escala micro (sitio arqueológico) se evaluó el modo de aprovisionamiento y uso de los recursos líticos, las estrategias tecnológicas y de subsistencia y la funcionalidad del sitio. En la escala meso (región) se discutió la importancia de rasgos del paisaje y de la localización de fuentes en la estructuración de la movilidad y el asentamiento de las sociedades humanas. En la escala macro se indagó sobre el modo en que estos sectores cordilleranos y de uso estacional se conectaron con áreas de ambas vertientes andinas. Mediante diversas herramientas de análisis espaciales en sistema de información geográfica (SIG) se evaluó la accesibilidad a fuentes, los costos de traslado (medidos en tiempo y distancia), la distribución espacial de muestras arqueológicas (Kernel Density Estimation y análisis falloff) y el acceso a las áreas de estudio desde enclaves importantes dentro del proceso de poblamiento regional.

Los estudios tecnológicos (Castro et al., 2014, Cortegoso et al., 2017, Durán et al., 2006, Lucero, 2015, entre otros) se enfocaron desde una perspectiva organizacional (sensu Nelson, 1991) y sistémica (sensu Ericson, 1984). El registro lítico se analizó tecno-morfológicamente (sensu Aschero, 1983) y se combinó con estudios de secuencias reductivas ${ }^{1}$ (sensu Cortegoso, 2008). Las

\footnotetext{
${ }^{1}$ Adquisición (fragmentos naturales o nódulos «potencialmente» susceptibles de ser tallados), Preparación (núcleos y productos indiferenciados), Talla Primaria (lascas externas), Talla Secundaria (lascas internas), Formatización (micro y
} 
materias primas se clasificaron macroscópicamente y se seleccionaron muestras geológicas y arqueológicas para análisis petrográficos de cortes de lámina delgada que permitieron hacer caracterizaciones precisas y, en algunos casos, aproximar la procedencia de los recursos.

\section{Cuenca alta del río de Las Taguas, Noroeste de San Juan $\left(29^{\circ} \mathrm{S}\right)$}

Este espacio abarca $380 \mathrm{~km}^{2}$ y corresponde a la Cordillera Principal de los Andes Áridos (Figura 2), ubicado entre el límite internacional argentino-chileno al oeste (5.500 msnm) y la Cordillera de la Ortiga al este (5.000 msnm). En el centro del área se encuentra el valle del río de Las Taguas (3.700 msnm). En el año 2006 se inició un programa de rescate y estudios arqueológicos (Durán y Cortegoso, 2010). Se relevaron y rescataron 29 sitios que incluyen campamentos semipermanentes de actividades múltiples, fuentes y canteras líticas. La amplia secuencia ocupacional se caracteriza por ocupaciones de cazadoresrecolectores entre 9980 y 7520 años cal. AP, de posibles grupos transicionales entre 5810 y 4800 cal. AP y de pastores plenos entre3990y 510 años cal. AP en adelante (Lucero et al., 2017, entre otros).

Las investigaciones se iniciaron con la elaboración de una carta geológica en una escala de 1:100.000, adaptada al marco de indagación arqueológica (Castro et al., 2014). Los análisis de SIG indicaron que las brechas hidrotermales y las tobas riolíticas cristalinas son los recursos litológicos de mayor superficie. Se detectaron siete fuentes primarias (ARQ-C15, ARQ-C14, Amarillos, Potrerillos A, Potrerillos $B$, Taguas y Turbio) y tres secundarias (Amarillos, Guanaco Zonzo y Taguas). Cinco fuentes tienen talleres líticos que evidencian su uso antrópico y cuatro se consideran canteras potenciales. El análisis espacial SIG indicó que todas las fuentes son locales, es decir con costos de accesibilidad menores a 8 hs (Lucero et al., 2017). El ultramicrolascas), Instrumentos (artefactos formatizados) y Mantenimiento (lascas de reactivación de filos). análisis de secuencias reductivas de fuentes y canteras ( $\mathrm{N}=2221)$ y de sitios de actividades múltiples ( $\mathrm{N}=30886)$ evidenció que durante los casi 8500 años de ocupación del área, por un lado, se mantuvieron sistemas de producción lítica secuenciales (sensu Ericson, 1984) y, por otro lado, la procedencia de los recursos fue diferencial (Castro et al., 2014; Lucero et al., 2017, entre otros). Las rocas locales predominan en toda la secuencia ocupacional ya que los grupos utilizaban estos recursos para aprovisionarse demateria prima y transportarla a las bases residenciales. El análisis sobre los costos de accesibilidad a las fuentes y calidad de los recursos, indicó que los cazadoresrecolectores con alta movilidad residencial explotaron las fuentes locales mejor rankeadas. Al contrario, los grupos con economías mixtas y movilidad trashumante diversificaron el uso de fuentes locales incluyendo las de menor ranking y trasladaron a este espacio recursos foráneos. Estos cambios en la explotación de recursos se relacionan con mayor recurrencia ocupacional y con la integración del área a circuitos de movilidad periódicos y predecibles.

\section{Bosque de Darwin y Los Colorados, Noroeste de Mendoza $\left(32^{\circ} \mathrm{S}\right)$}

El área de estudio contiene dos sectores con bosques fósiles: el Bosque de Darwin, localizado en la formación geológica Paramillos (94 ha) y un sector a $3 \mathrm{~km}$ de distancia en la formación geológica Los Colorados (49 ha). Se ubica en la precordillera de Mendoza (2700 msnm), al Este del valle longitudinal de Uspallata (Figura 3). Los trabajos multidisciplinares geoarqueológicos favorecieron la descripción detallada de las formaciones geológicas que contienen los sectores con troncos fósiles, la localización de los especímenes, los procesos que afectan su calidad para la talla y la evidencia de su explotación prehistórica (Cortegoso et al., 2017).

En el Bosque de Darwin se observó una ausencia casi absoluta de troncos en posición de vida, quedando las improntas sobre formaciones rocosas (Cortegoso et al.,
Figura 2. Fuentes de materia prima lítica en el Valle del río de Las Taguas (Noroeste de la provincia de San Juan).

Figure 2. Lithic raw material sources from the Las Taguas River valley Northwestern San Juan province).

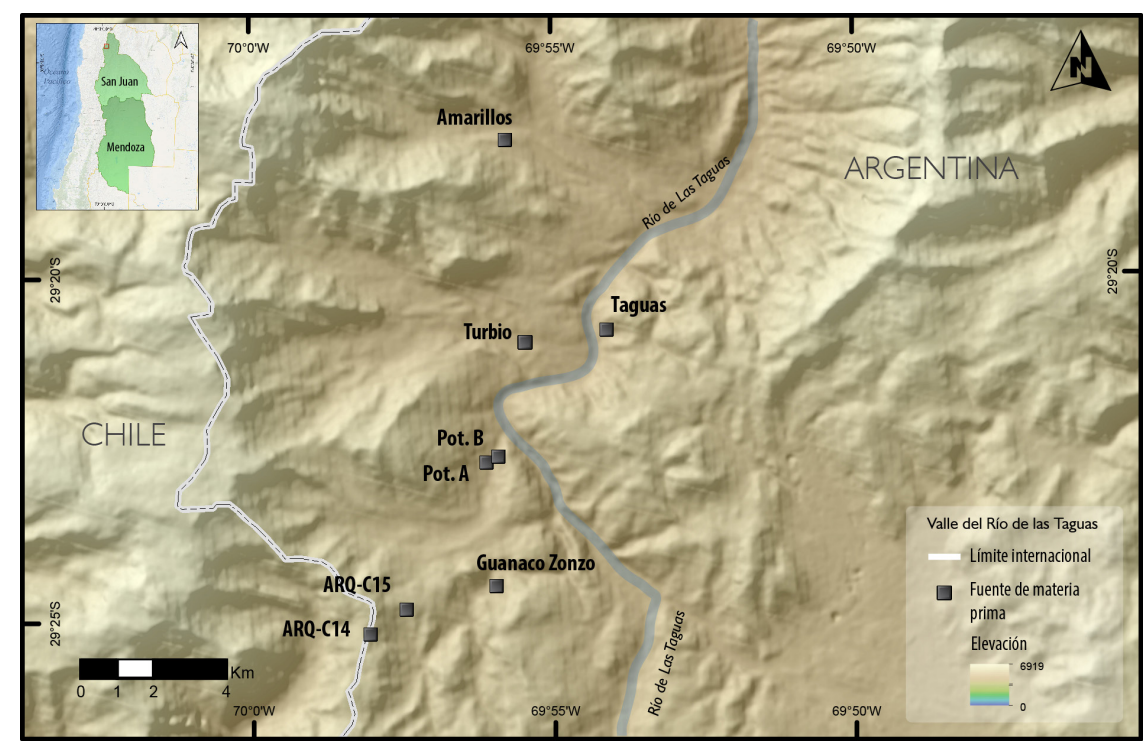

ISSN 1852-060X (impreso) / ISSN 1852-4826 (electrónico) 


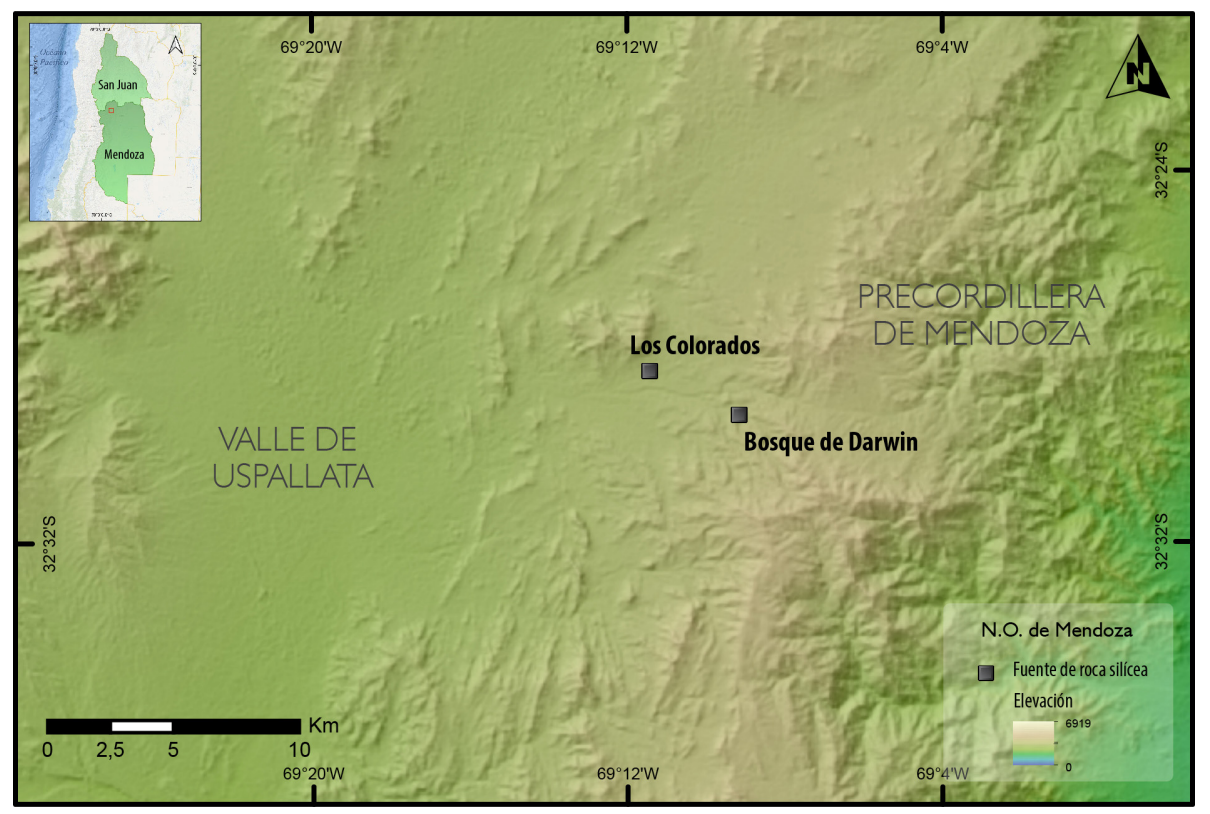

Figura 3. Fuentes de materia prima lítica en el Bosque de Darwin y Los Colorados (Noroeste de la provincia de Mendoza).

Figure 3. Lithic raw material sources from Bosque de Darwin and Los Colorados (Northwestern Mendoza province).

2017, p.9, figura 4). El análisis petrográfico demostró la existencia de procesos de génesis volcánicos e hidrotermales que alteraron rocas previas (sedimentarias y tobas) y la flora del lugar, resultando en agregados microgranulares de buena calidad para la talla. Se identificaron especímenes desplazados y dispersiones primarias $(\mathrm{N}=15)$, con asociación de restos de talla en pequeñas concentraciones de calidades muy variables según su grado de silicificación. Se identificó un taller lítico asociado al Bosque de Darwin con abundante registro arqueológico $\left(\mathrm{N}=762^{2}\right.$, en transectas que cubrieron $600 \mathrm{~m}^{2}$ equivalente al $11 \%$ del sector con restos de talla), indicando un uso casi exclusivo de las rocas disponibles en ambos sectores de bosques fósiles. Las actividades extractivas remiten fundamentalmente a las primeras etapas de selección y desbaste inicial de las piezas líticas. También se registraron dos fragmentos de obsidiana alóctona. En la formación Los Colorados se localizaron dispersiones primarias y troncos desplazados $(\mathrm{N}=14)$ con evidencias de canteo (Cortegoso et al., 2017, p.11, figura 5).

Los análisis espaciales SIG reflejaron que las rutas más eficientes son las que conectan a las fuentes con los sectores occidentales de la precordillera (Cortegoso et al., 2017, p.13, figura 7). Allí se ubica el sitio Agua de la Cueva que registra en sus contextos tempranos (ca. 7400 años AP) instrumentos con formatización bifacial retomados y elaborados sobre las materias primas de estas fuentes. Por otra parte, contextos tardíos (ca. 1000 años AP) del sitio Vaquería, localizado en el piedemonte y más alejado de las fuentes, registran formatización y mantenimiento de puntas de proyectil sobre este tipo de materias primas silíceas (ver citas en Cortegoso et al., 2017). Los bosques fósiles y el sitio Vaquerías se ubican en espacios vinculantes entre tierras altas y bajas transitados durante todo el Holoceno por grupos con

\footnotetext{
2 Las piezas corresponden a nódulos «potencialmente» susceptibles de ser tallados, núcleos, productos indiferenciados, lascas con talón y artefactos formatizados.
}

distintas estrategias de subsistencia. Es probable que con el tiempo la explotación de estas fuentes haya sido más recurrente desde el valle de Uspallata. En consecuencia se habría potenciado el uso de la ruta que conecta con el cerro El Tunduqueral. Este valle posee condiciones ambientales y altitud adecuada para los asentamientos residenciales anuales y tiene un registro arqueológico que testimonia la existencia de distintos tipos de sitios durante el Holoceno. Elementos diversos dan cuenta de la compleja escala de interacción entre las sociedades a ambos lados de la cordillera en tiempos tardíos (ver citas en Cortegoso et al., 2017). Así, la ocupación efectiva del valle de Uspallata pudo haber estado acompañada por el desarrollo de sistemas recurrentes de explotación de los recursos de estas fuentes (Cortegoso et al., 2017).

\section{Laguna del Diamante, Centro-Oeste de Mendoza $\left(34^{\circ} \mathrm{S}\right)$}

Esta área de estudio cubre un espacio de $20 \times 16 \mathrm{~km}$ (Figura 4). Se encuentra en la Cordillera del Límite de los Andes Áridos (3300msnm) e incluye la Reserva Natural Laguna del Diamante, emplazada en el interior de una caldera volcánica de colapso. Los eventos eruptivos que dieron origen a la caldera, hace $450 \mathrm{ka}$, expulsaron ignimbrita que cubrió tanto la ladera argentina como la chilena (Stern et al., 1984). Los datos radiocarbónicos indican que la ocupación humana en el interior de la Laguna se remonta a los últimos 2000 años AP (Durán et al., 2006). Esta cronología marca el inicio de procesos culturales divergentes al norte y sur de los $34^{\circ}$ $\mathrm{S}$ : al norte, sociedades con economías mixtas; al sur, cazadores-recolectores. En el área se registraron 17 sitios arqueológicos, entre ellos dos fuentes de obsidiana.

El estudio sobre procedencia de obsidianas es uno de los temas centrales de estas investigaciones. Se efectuaron diversos estudios traza (NAA, FRX y LA-ICP-MS) a más de 
Figura 4. Fuentes de materia prima lítica en la Reserva Natural Laguna del Diamante (CentroOeste de la provincia de Mendoza).

Figure 4. Lithic raw material sources from the Laguna del Diamante Natural Reserve (Central Western Mendoza province).

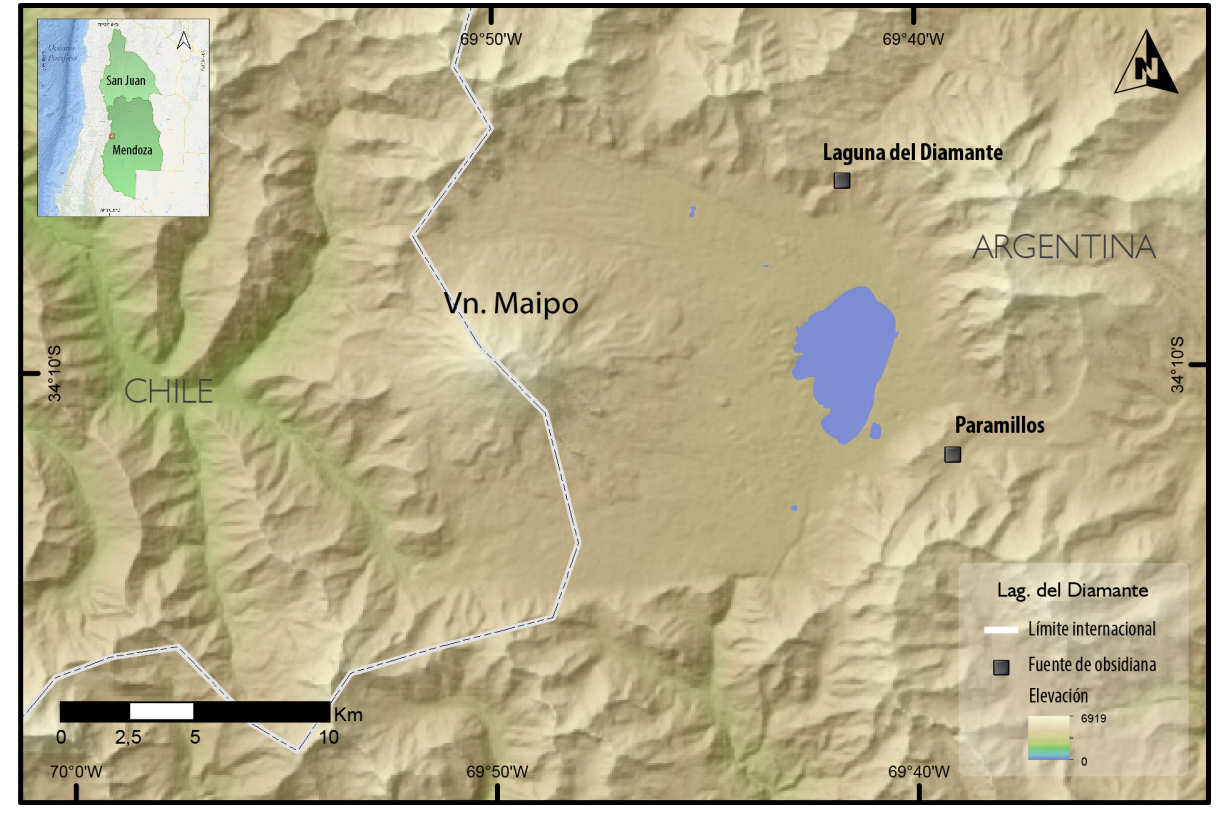

En el caso de áreas de fuentes con variada extensión y ubicuidad (valle del Taguas, por ejemplo), fue de particular relevancia la realización de la carta geológica a escala arqueológica que permitió refinar el grado de resolución sobre la información litológica de los afloramientos. Los análisis petrográficos realizados en todas las áreas, en muestras geológicas y arqueológicas, fueron fundamentales para la caracterización de la oferta y el estudio de la génesis de los recursos líticos. También proporcionaron una aproximación más específica a las fuentes, como el caso de la explotación del bosque fósil de Darwin, cuyos cortes de lámina delgada muestran las estructuras orgánicas que remiten a estas formaciones. La asignación de artefactos a las fuentes por medio de estudios geoquímicos combinado con SIG, como el caso de la Lagua del Diamante, está permitiendo discutir no sólo la extensión de las fuentes cordilleranas sino también las áreas desde las que se accede y explotan los ambientes estacionales en las que se ubican. Los trabajos realizados en este tipo de espacios reafirman la necesidad de considerar la topografía como un factor crítico en la movilidad. Los análisis espaciales SIG indicaron la pertinencia de calcular costos de accesibilidad a fuentes en términos de tiempo y distancia.

El desarrollo de estas investigaciones permitió evaluar cambios y continuidades en el uso de recursos líticos vinculados a variaciones en las estrategias de subsistencia y movilidad, caracterizar y modelar el acceso a fuentes de acuerdo con un ranking de costo y calidad e identificar vectores dominantes de acceso a ambientes de altura. Así, el estudio sobre las primeras etapas productivas de la tecnología lítica es una importante vía de acceso para indagar sobre los principales cambios en las estrategias humanas desde distintas escalas espaciales. Frente a la creciente importancia a nivel mundial de los estudios de fuentes, aún se deben robustecer con la implementación de enfoques multidisciplinarios que utilicen y generen datos 
empíricos de naturaleza variada para la caracterización de las mismas. El estudio de toda fuente lítica debe iniciarse con una caracterización estructural que informe sobre la génesis de las rocas, la extensión de las fuentes, la dispersión de los recursos, los agentes naturales y culturales que modifican tal disponibilidad, entre otros. Este conocimiento es un requisito imprescindible antes de realizar inferencias culturales sobre el uso de las canteras, el tipo de aprovisionamiento y los rangos de movilidad.

\section{Agradecimientos}

Las investigaciones presentadas se llevaron en el marco de proyectos financiados por la Agencia Nacional de Promoción Científica y Tecnológica de Argentina (PICT 0940) y por el Consejo Nacional de Investigaciones Científicas y Técnicas (PIP 2015-2018).El trabajo de síntesis fue presentado en el simposio "El estudio de los conjuntos líticos en las áreas de aprovisionamiento: variabilidad y formas de abordaje desde el análisis técnicotipológico", desarrollado en el $1^{\text {er }}$ Congreso Argentino de Estudios Líticos en Arqueología (CAELA) durante el año 2018.

Mendoza, 15 de abril de 2019

\section{Referencias citadas.}

Aldenderfer, M. S. (2008). High elevation foraging Societies. En H. Silverman y W. H. Isbell (Eds.), Handbook of South American Archaeology (pp. 131-143). New York: Springer.

Aschero, C. (1983). Ensayo para una clasificación morfológica de artefactos líticos aplicada a estudios tipológicos comparativos. Apéndices $A-C$. Revisión. Cátedra de Ergología y Tecnología (FFyL-UBA). Buenos Aires. Ms.

Castro, S., Gasco, A., Lucero, G.,Cortegoso, V., y Durán, V. (2013). Mid-Holocene hunters and herders of southern cordillera, Northwestern Argentina.Quaternary International, 307, 96-104.

Castro, S., Lucero, G., Cortegoso, V., y Winocur, D. (2014). Fuentes de aprovisionamiento de materia prima y sistemas de producción lítica en Los Andes (Noroeste de San Juan, Argentina). Relaciones de la Sociedad Argentina de Antropología, XXXIX(2), 365-386.

Cortegoso, V. (2008). Disponibilidad de recursos líticos en el Noroeste de Mendoza: cambios en la organización tecnológica en la cuenca del río Blanco. Cazadores-Recolectores del Cono Sur. Revista de Arqueología, 3, 95-112.

Cortegoso, V. (2005). Mid-Holocene hunters in the Andes Mountains: environment, resources and technological strategies. Quaternary International, 132, 71-80.

Cortegoso, V. (2006). Comunidades agrícolas en el Valle de Potrerillos (NO de Mendoza) durante el Holoceno tardío: organización de la tecnología y vivienda. Intersecciones en Antropología, 7, 77-95.

Cortegoso, V., Barberena, R., Durán, V., y Lucero, G. (2016). Geographic vectors of human mobility in the Andes (34-36 $\mathrm{S}$ ): Comparative analysis of "minor" obsidian sources. Quaternary International, 422, 81-92.
Cortegoso, V., Durán, V., y Gasco, A. (Eds.) (2014). Arqueología de Ambientes de Altura de Mendoza y San Juan (Argentina). EDIUNC. Mendoza.

Cortegoso, V., Lucero, G., Castro, S., y Winocur, D. (2017). Bosques fósiles y tecnología humana: la explotación de materias primas líticas en el Bosque de Darwin, Paramillos (Argentina). American Antiquity, 28(3), 317-336.

De Francesco, A. M., Barca, D., Bocci, M., Cortegoso, V., Barberena, R., Yebra, L., y Durán, V. (2018). Provenance of obsidian artifacts from the Natural Protected Area Laguna del Diamante (Mendoza, Province Argentina) and upper Maipo valley (Chile) by LA-ICP-MS method. Quaternary International, $468,134-140$.

Dincauze, D. (2000). Environmental Archaeology. Principles and Practice. Cambridge: Cambridge University Press.

Durán, V., y Cortegoso, V. (2010). Programa de Rescate y Estudios Arqueológicos del Área del Proyecto Minero Pascua Lama (Argentina). Informe Final (Período 2006-2010).

Durán, V., Neme, G., Cortegoso, V., y Gil, A. (2006). Arqueología del área natural protegida Laguna del Diamante (Mendoza, Argentina). En V. Durán y V. Cortegoso (Eds.), Arqueologia y ambiente de áreas naturales protegidas de la provincia de Mendoza (Volumen especial de Anales de Arqueología y Etnología, 61, pp. 81-134). Mendoza: CEIDER.

Ericson, J. (1984). Toward the analysis of lithic production systems. En J. Ericson y B. Purdy (Eds.), Prehistoric quarries and lithic production (pp. 1-9). New York: Cambridge University Press.

Giesso, M., Durán, V., Neme, G., Glascock, M., Cortegoso, V., Gil, A., y Sanhueza, L. (2011). A study of obsidian source usage in the Central Andes of Argentina and Chile. Archaeometry, $53(1), 11-21$.

Ingbar, E. (1994). Lithic material selection and technological organization. En P. J. Carr (Ed.), The Organization of North American Prehistoric Chipped Stone Tool Technologies (pp. 45-56). Michigan: Archaeological Series 7. International Monographs in Prehistory.

Lucero, G. 2015. Biogeografía y paleoecología humana de tierras altas: subsistencia y tecnología en el valle del río de Las Taguas (departamento de Iglesia, provincia de San Juan).Tesis Doctoral Inédita. Facultad de Filosofía y Letras, UNCuyo. Mendoza.

Lucero, G., Castro, S., y Cortegoso, V. (2017). Tecnología lítica de cazadores y pastores andinos: cambios y continuidades en la explotación de recursos líticos durante el Holoceno en el NO de San Juan. Revista del Museo de Antropología, Suplemento Especial 1, 65-74.

Nami, H. (1992). El subsistema tecnológico en la confección de instrumentos líticos y la explotación de los recursos del ambiente: una nueva vía de aproximación. Shincal, 2, 33-53.

Nelson, M. (1991). The Study of Technological Organization. Archaeological Method and Theory, 3, 57-100.

Stern, C. R., Amini, H., Charrier, R., Godoy, E., Herve, F., y Varela, J. (1984). Petrochemistry and age of rhyolitic pyroclastic flows which occur along the drainage valleys of the rio Maipo and rio Cachapoal (Chile) and the rio Yaucha and Papagayo (Argentina). Revista Geológica de Chile, 23, 39-52. 